\title{
EFFICIENCY OF ORTHOPAEDIC BRACE WITH LASER ON KNEE JOINT FUNCTION, PAIN AND SWELLING
}

\author{
Tomas Indriulionis ${ }^{1,2}$, Juozas Raistenskis ${ }^{1,2,5}$, Kęstutis Saniukas ${ }^{1,2,5}$, Rūta Garšvienè2, \\ Viktorija Zacharenko², Viktoras Vaičikauskas ${ }^{3}$, Sigitas Kamandulis ${ }^{4}$, Audrius Sniečkus ${ }^{4}$, \\ Vida Janina Česnaitienè $\dot{ }^{4}$, Dovilè Kielè ${ }^{4}$, Neringa Baranauskiené $\dot{e}^{4}$, Nerijus Eimantas ${ }^{4}$, \\ Rima Solianik $^{4}$, Mantas Mickevičius ${ }^{4}$, Albertas Skurvydas ${ }^{4}$, Vytautas Streckis $^{4}$, \\ Renata Žumbakytė-Šermukšniene் ${ }^{4}$ \\ Vilnius University Hospital Santariškiu Klinikos ${ }^{1}$, Vilnius, Lithuania \\ MT Sprendimai ${ }^{2}$, Vilnius, Lithuania \\ Centre for Physical Sciences and Technology, Vilnius, Lithuania \\ Lithuanian Sports University ${ }^{4}$, Kaunas, Lithuania \\ Vilnius University, Vilnius, Lithuania
}

\begin{abstract}
Background. The effectiveness of outpatient mobile laser intervention on specific treatment goals has not been fully explored. Thus, the aim of the study was to examine the effect of orthopaedic knee brace with laser on knee joint function, pain and swelling.

Methods. Volunteers were divided into two experimental groups: chronic pain $(n=20$, knee pain $>6$ months $)$ and acute pain ( $n=20$, knee pain $<1$ week), and one control ( $n=20$, placebo) group. Subjects in all groups were immobilized in a knee brace for 4 weeks. In addition, the experimental groups underwent low-intensity laser therapy, whereas control group underwent pseudo-laser therapy, every second day for $10 \mathrm{~min}$. The sequences of the tests were as follows: (a) clinical assessments and evaluation of knee extension and flexion amplitude, swelling, Lysholm subjective assessment questionnaire, VAS pain scale, (b) evaluation of balance using Kistler platform, (c) muscle peak torque and isometric torque variability assessment using isokinetic dynamometry. All tests were performed twice - before and after intervention.

Results. Knee brace with laser is effective treatment for the reduction of pain, swelling, and recovering of daily functions $(p<.05)$. Operation of the complex device is particularly effective in treating knee pain in the acute period $(p<.05)$.

Conclusions. Knee brace with laser therapy in the rehabilitation period ensures faster healing of patients compared to wearing only knee brace, particularly in acute pain, while muscle strength and proprioception remains depressed.
\end{abstract}

Keywords: mobile laser therapy, range of motion, swelling, muscle strength, proprioception.

\section{INTRODUCTION}

$\mathrm{K}$ nee joint have been most commonly injured joint in athletes (Bollen, 2000). In most cases the most vulnerable are anterior cruciate and lateral ligaments, meniscus, and knee cartilage. Injuries cause pain, swelling, decreased muscle strength and range of motion (ROM), which leads to chronic alterations such as arthropathy or chondropathy. Rehabilitation treatment is applied to cure the inflammatory processes and fully restore knee function. 


\begin{tabular}{|l|c|c|c|c|}
\hline & $\begin{array}{c}\text { All } \\
\text { subjects } \\
(\boldsymbol{N = 6 0 )}\end{array}$ & $\begin{array}{c}\text { Control } \\
\text { group } \\
(\boldsymbol{n}=\mathbf{2 0})\end{array}$ & $\begin{array}{c}\text { Experimental chronic } \\
\text { pain group } \\
(\boldsymbol{n}=\mathbf{2 0})\end{array}$ & $\begin{array}{c}\text { Experimental acute } \\
\text { pain group } \\
(\boldsymbol{n}=\mathbf{2 0})\end{array}$ \\
\hline Age, years (mean & 35.3 & 40.6 & 22.9 & 31.3 \\
and $(S D))$ & $(13.8)$ & $(18.0)$ & $(10.9)$ & $(10.7)$ \\
\hline Men & 38 & 15 & 11 & 12 \\
Women & 22 & 5 & 9 & 8 \\
\hline Injured left leg & 26 & 10 & 10 & 6 \\
Injured right leg & 34 & 10 & 10 & 14 \\
\hline
\end{tabular}

One of the most effective rehabilitation methods is laser therapy. Therapeutic effect of stationary (uninstalled in orthopaedic devices) low-frequency laser on the acceleration of healing of tissues has been substantially examined (Hegedus, Viharos, Gervain, \& Gálfi, 2009; Peter et al., 2011; Walsh, Baxter, \& Allen, 2000). The effect of low-intensity laser radiation is anti-inflammatory, anaesthetic, regenerative and promoting regional blood circulation and microcirculation (Bjordal, Couppé, Chow, Tunér, \& Ljunggren, 2003). Laser therapy stimulates the production of collagen, activates the metabolism, changes the DNA synthesis and improves the functions of damaged nerve (van Middelkoop et al., 2011; Rola, Doroszko, \& Derkacz, 2014).

At present, little is known about mobile devices with laser radiation aiming at effective improvement of muscle functions. Mobile laser may reduce treatment costs since the use of fixed installations necessitates the patient to attend the clinic for the procedures. The latter is a waste of time for both the patient and the clinic staff. Mobile devices enable the procedures to be performed at home or in any location convenient for the patient. However, the use of a mobile device requires some skills and knowledge from the patient and therefore it remains unclear whether the treatment effect is comparable to the one demonstrated previously with stationary ones (Bjordal at al., 2008; van Middelkoop et al., 2011). Consequently, the aim of the study was to examine whether the mobile orthopaedic knee brace with laser is effective for knee joint function improvement, swelling, and acute or chronic pain reduction.

\section{METHODS}

Subjects. The study included 60 subjects with a doctor appointed knee brace with laser effects by the indications. The subjects' data can be viewed in the Table. In the case of chronic pain, the experimental group $(n=20)$ included subjects who met the following criteria: knee pathology with arthropathy, systemic connective tissue diseases, soft tissue diseases, osteopathy, chondropathy, sore knee for at least 6 months. In the case of acute pain, the experimental group (EA; $n=20$ ) included subjects who met the following criteria: experienced knee injuries, knee surgery after traumatic injuries (knee ligament tear, meniscus tear intraarticular fractures, patella injuries, etc.). Subjects in control groups met the criteria of chronic $(n=10)$ and acute pain $(n=10)$.

Each subject read and signed a written informed consent form consistent with the principles outlined in the Declaration of Helsinki. Kaunas Regional Bioethics Committee approved this study.

Experimental protocol. During the study, the subjects underwent the combined treatment using immobilization (knee brace) and laser therapy. The stages of the testing were as follows: (a) clinical assessments and evaluation of active knee joint ROM, swelling, Lysholm subjective assessment questionnaire, VAS pain scale, (b) evaluation of balance using Kistler platform, (c) muscle peak torque and isometric torque variability assessment using isokinetic dynamometry. All tests were performed twice: before and after wearing the knee brace with laser device for four weeks, while applied laser therapy at home was used every second day for $10 \mathrm{~min}$. Control group subjects had laser imitation instead of real laser in the knee brace. Before each test trial, subjects received standardized verbal instructions and completed from two to three familiarization trials using submaximal efforts.

Knee brace with laser. The construction consists of commercial visible or infrared laser diodes installed in the knee brace bodies and connected to a power source by flexible wires (Figure 1). The device uses $808 \mathrm{~nm}$ infrared laser 
diodes and the visible area $650 \mathrm{~nm}$ wavelength diodes. Laser type and operating mode is selected according to the nature of the pathology. According to the nature of pain, three types of braces with four embedded laser diodes are used. The electric circuit of the device is powered by a $3.7 \mathrm{~V} \mathrm{Li-ion}$ battery. Diode operating voltage is up to 2.2 volts. According to the doctor's recommendations, laser diodes are fitted at the specified locations on the knee brace hinge. Power supply is a separate small block which is also fixed to the brace.

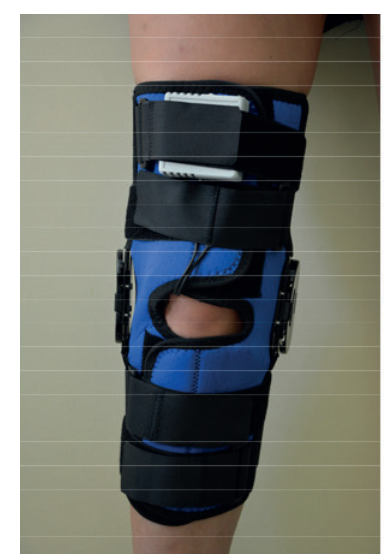

Figure 1. Knee brace with laser

Maximal voluntary isometric and concentric torque. The maximal voluntary isometric contraction peak torque (MVIC) of the knee extensor and flexor muscles of the involved leg (uninvolved MVIC in subjects in acute pain group) was measured using an isokinetic dynamometer (System 3; Biodex Medical Systems, Shirley, New York). The subjects sat in an upright position on the Biodex Dynamometer Accessory Chair and were secured using thigh, pelvic and torso straps to minimize extraneous body movements. Gravity correction was done using the torque exerted on the dynamometer resistance adapter when the knee was in a relaxed state. Calibration of the Biodex dynamometer was performed according to the manufacturer's service manual prior to each testing session. Each subject was required to fold their arms across his chest and was given verbal encouragement and visual feedback from the Biodex computer monitor to facilitate maximaleffort contractions.

Maximal isometric and concentric strength of the quadriceps and hamstring muscles were investigated using 1-minute rest periods between series of measurements. Isometric trials were completed at a knee angle of $70^{\circ}\left(0^{\circ}\right.$ : knee fully extended). Subjects completed two 3-5-second maximal isometric knee extensions while they were asked to produce their maximal force as fast as possible. For the concentric isokinetic trials, ROM was $80^{\circ}$, from $100^{\circ}$ to $20^{\circ}$ knee flexion, and vice versa. Concentric tests were performed at two angular velocities: $60 \%$ second and $180 \%$ second. Subjects were asked to complete the full ROM during three consecutive extension-flexion trials.

Isometric torque variability test. Isometric torque variability was established during a 20 -s submaximal isometric contraction. Target torque with chronic pain group participants was equal to $20 \%$ of MVIC involved legs, subjects with acute pain group $-10 \%$ of uninvolved legs. Subjects were asked to perform the tasks as accurately as possible with visual feedback, i.e. the subjects were able to see the torque signal. Knee extension and flexion torques of the damaged leg during 3-20 s isometric contractions were analysed, and the coefficient of variation $(\mathrm{CV})$ was calculated as an indicator of motor output variability (Skurvydas et al., 2011). Knee extension and flexion variability was tested at $70^{\circ}$ knee joint angles. The rest interval between measurements was $45 \mathrm{~s}$. Previously published methodology (Skurvydas et al., 2011) was used in the study.

Subjective knee function evaluation. Lysholm questionnaire was used to evaluate the subjective functional state of the knee joint. Subjects subjectively assess their ability to carry out daily activities determined by the condition of the knee joint (Tegner \& Lysholm, 1985).

Pain. Assessment of pain was carried out on a visual analogue scale (VAS) scores where research participants subjectively evaluated perceived pain (from 0 to 10; 0 - almost no pain, 10 - unbearable pain) (Miyama \& Nosaka, 2007).

Inflammatory process assessment. In order to evaluate knee swelling and oedema as well as their pre-post dynamics, the circumference (girth) of the knee in three standardized points was measured with a tape measure: over the joint, at the patella, and below the knee.

Range of motion. Active knee joint ROM (flexion and extension) was measured using a universal goniometer. The subjects was positioned supine, with the hip and knee in the neutral position. The femur was stabilized to prevent rotation with a hand.

Balance. The balance of the subjects was assessed by the method of static posturography. 
Kistler force plate (Switzerland, Slimline System 9286) and computer equipment registering signals were used for this purpose. This measurement system is meant to explore human biomechanical parameters and used in research institutions. Balance is measured when the subject stood on the force plate for $60 \mathrm{~s}$. The position of the subject on the platform was as follows: eyes open, looking straight ahead; feet put together, arms down at the sides, palms inwards. The duration of registering the posturogram was $60 \mathrm{~s}$, sampling rate $-100 \mathrm{~Hz}$. We registered the changes in velocity of the centre of pressure (COP) displacement in the lateral and anterior-posterior directions. The registration of the posturogram was started when the subjects took the correct posture on the force plate. Two trials were performed; the best result of the second trial was used for assessment. The frequency of the registered signal was $50 \mathrm{~Hz}$.

Statistical analysis. The different outcome measures were verified for normal distribution using the Kolmogorov-Smirnov test. Descriptive data are presented as the mean $\pm S D$. The level of significance was set at $p<.05$. Paired Student's $t$ tests were used to test differences in outcome measures between the separate values. Statistical analyses were performed using the Statistical Package for Social Sciences (SPSS software version 11.0 Cary, NC, USA).

\section{RESULTS}

Peak torque values of muscle of knee extension and flexion. There were no statistically significant differences between pre and post intervention of knee extension $(p>.05$; Figure 2a) and flexion $(p>.05$; Figure $2 b)$ in MVIC in both control and experimental groups. However, concentric knee extension and flexion torque performed at angular velocities of $60 \%$ second post intervention increased in EC group $(p<.05)$, whereas torque performed at angular velocities $180 \%$ second changed insignificantly $(p>.05)$. In control group, concentric knee extension and flexion torques in both velocities remained unchanged $(p>.05)$.

Intraindividual variability in knee extension and flexion torque at submaximal level. The results showed that neither control nor EC or EA subjects experienced a significant effect $(p>.05)$
Figure 2. Mean values (coefficient of variation (CV)) of maximal isometric (MVIC) and concentric, performed at angular velocities $60 \%$ (CON 60) and $180 \%$ s (CON 180), peak torques for the knee joint in control and experimental chronic pain (EC) groups pre and post wear of brace. (a) knee extension; (b) knee flexion

Note. $* p<.05$ comparing to pre intervention value.

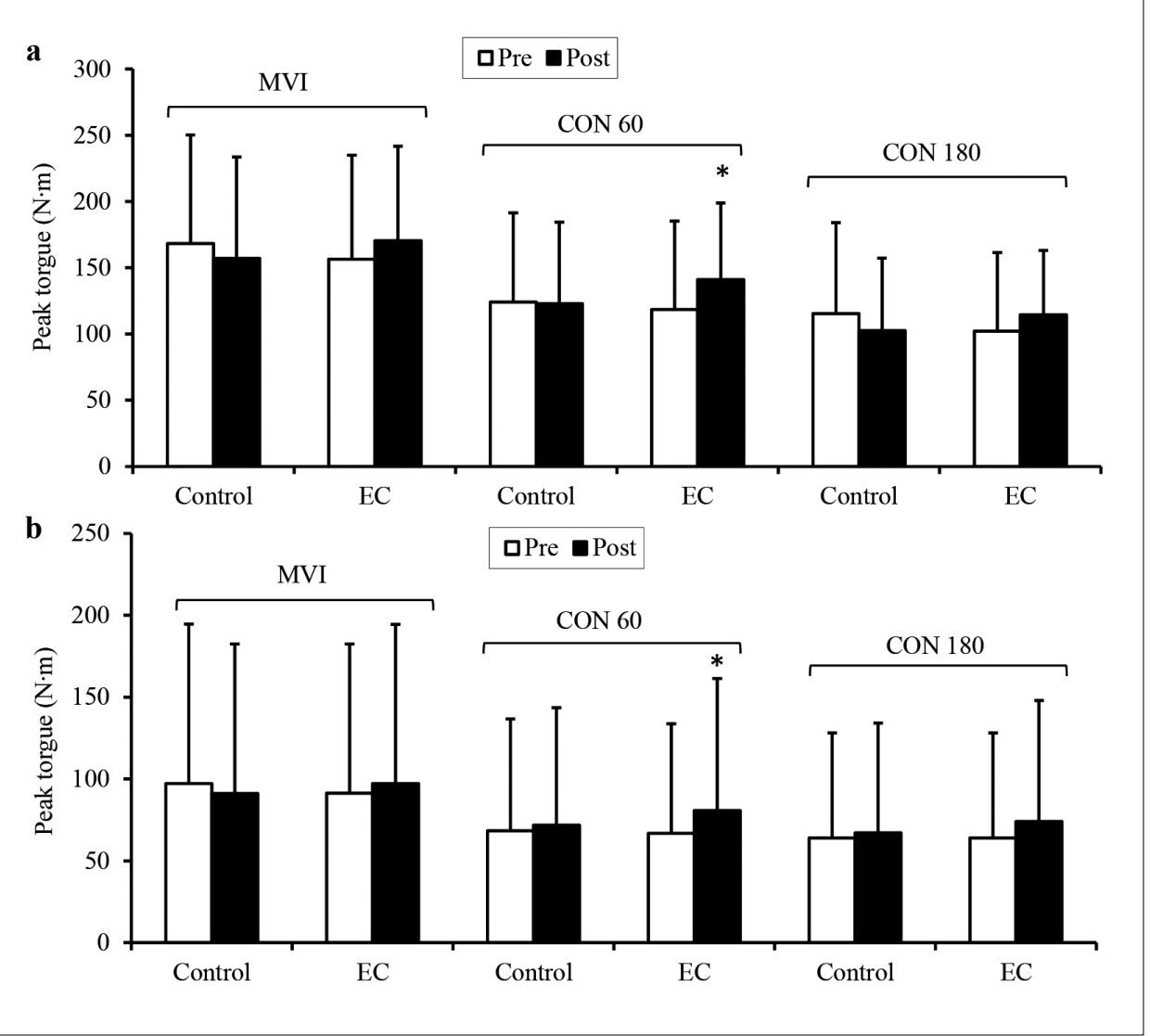



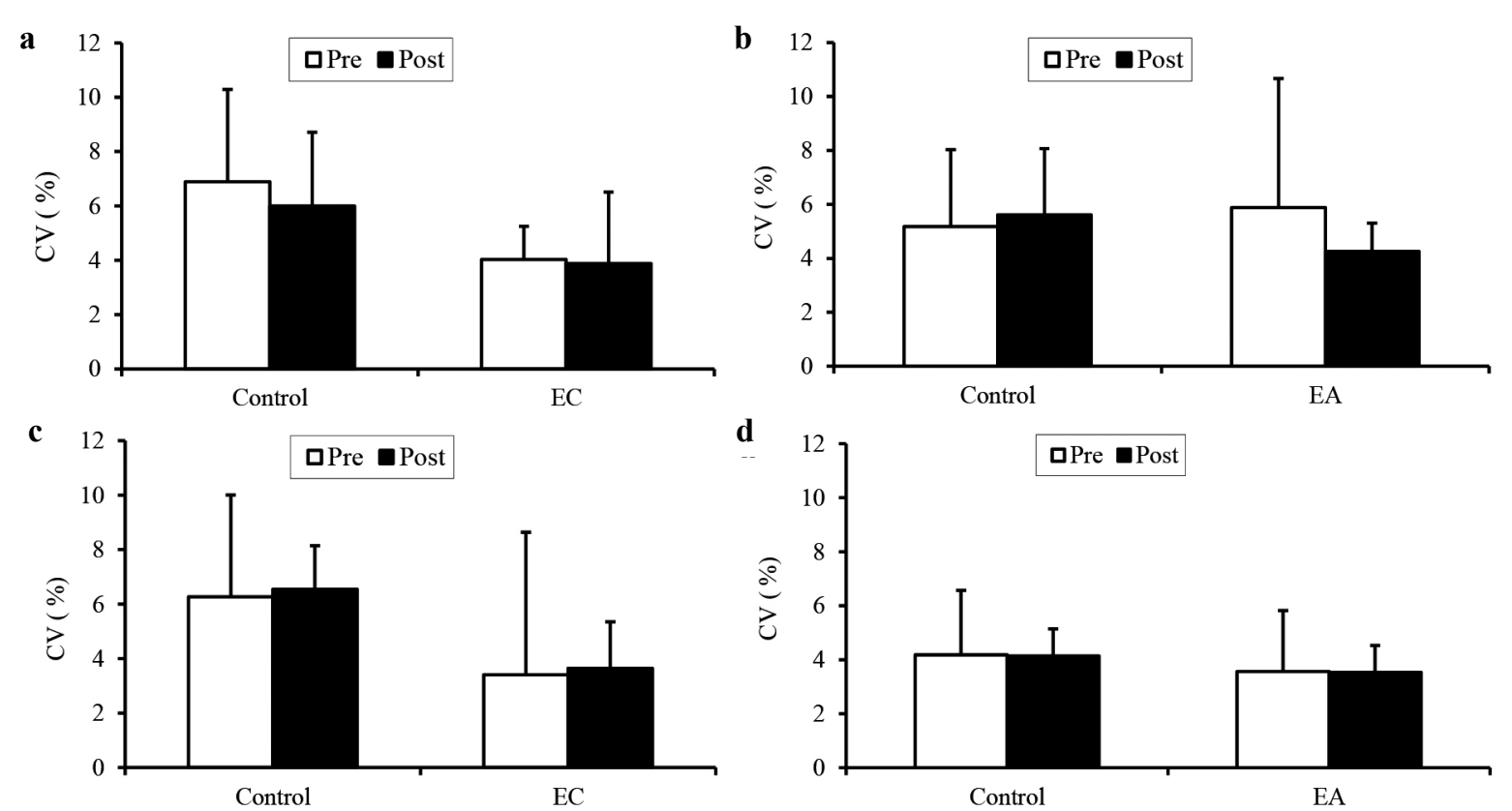

Figure 3. Mean values (coefficient of variation (CV)) of submaximal isometric torque variability in control, Experimental Chronic pain (EC) and Experimental Acute pain (EA) groups pre and post brace wear. (a and b) knee extension; (c and d) knee flexion

on knee extension and flexion torque variability (CV) after wearing a knee brace (Figure 3).

Knee function. Before wearing the Knee Brace Lysholm average mean scores in EA subjects were significantly lower than those in the control and EC groups of subjects, while control and EC did not differ significantly (Figure $4 ; p>.05$ ). Post Knee Brace wear score significantly increased in all groups $(p<.05)$. The highest increase $(p<$ $.05)$ was in EA group (43.4 points compared with control (9.6 points) and EC (19.4 points) subjects. Post Knee Brace wear average mean scores in experimental EC and EA group subjects were significantly higher than those in the control group subjects $(p<.05)$.

Pain. The initial values of pain among all groups (control, EC and EA) differed significantly (Figure 5; $p<.05$ ). After the Knee Brace wear pain assessment scores significantly decreased in all groups $(p<.05)$ and did not differ among themselves. Decrease (pre-post Knee Brace wear) was higher in the EA group compared to that in the control group and the EC $(p<.05)$. EA and EC group mean change scores did not differ significantly.

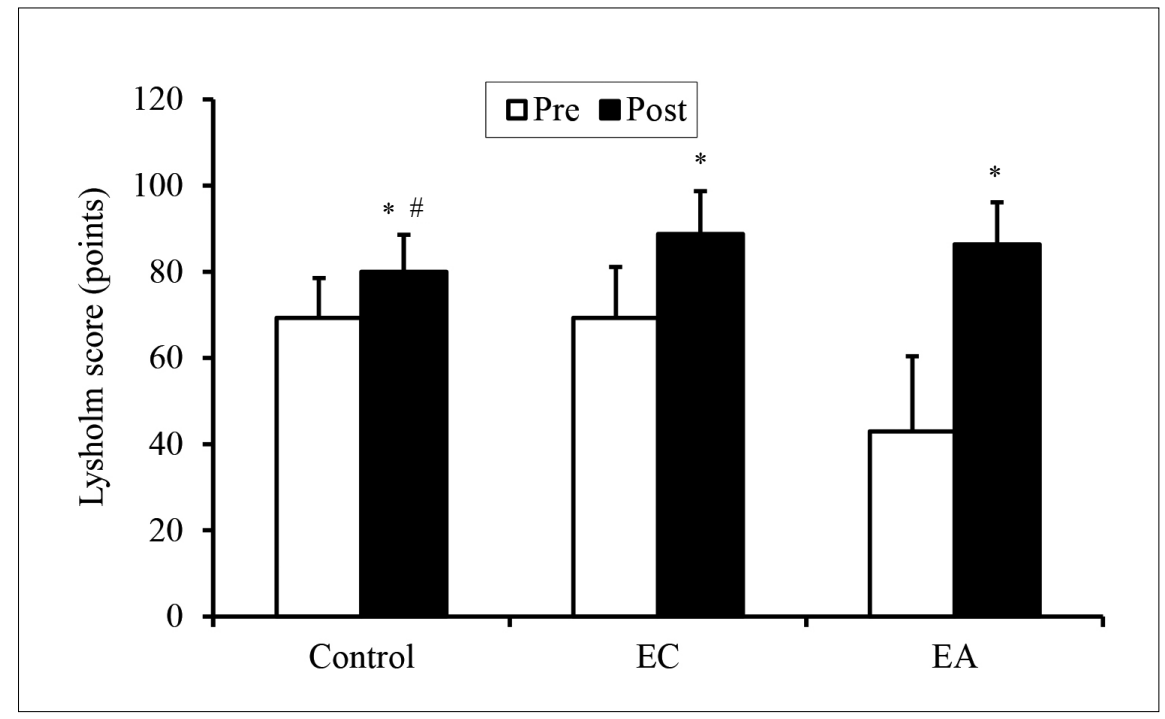

Figure 4. Mean values (coefficient of variation (CV)) of Lysholm Knee score in Control, Experimental Chronic pain (EC) and Experimental Acute pain (EA) groups pre and post knee brace wear

Note. ${ }^{*} p<.05$ comparing to pre intervention value, $\# p<.05$ control compared to EC and EA groups. 
Figure 5. Mean values (coefficient of variation (CV)) of Visual Analogue Pain Scale (VAS) score of Control, Experimental Chronic pain (EC) and Experimental Acute pain (EA) groups pre and post knee brace wear

Note. $* p<.05$ comparing to pre intervention value.

Figure 6. Mean values (coefficient of variation $(\mathrm{CV})$ ) of the range of motion (ROM), (a) flexion; (b) extension of Control, Experimental Chronic pain (EC) and Experimental Acute pain (EA) groups pre and post knee brace wear
Note. ${ }^{*} p<.05$ comparing to pre intervention value.

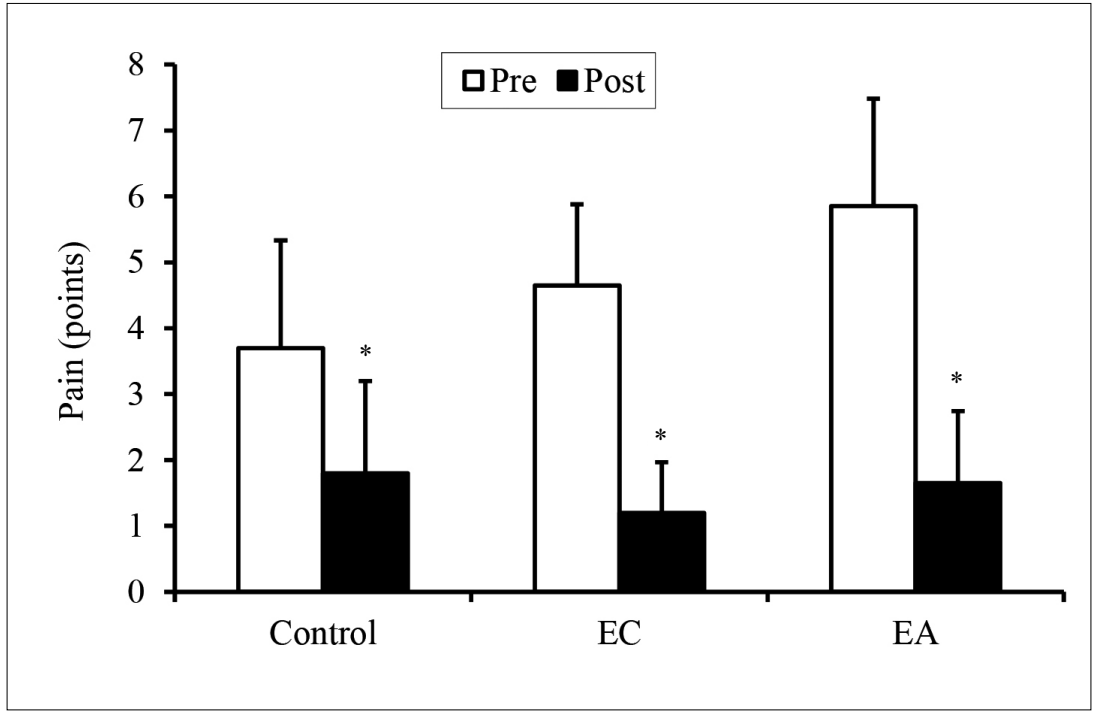

a

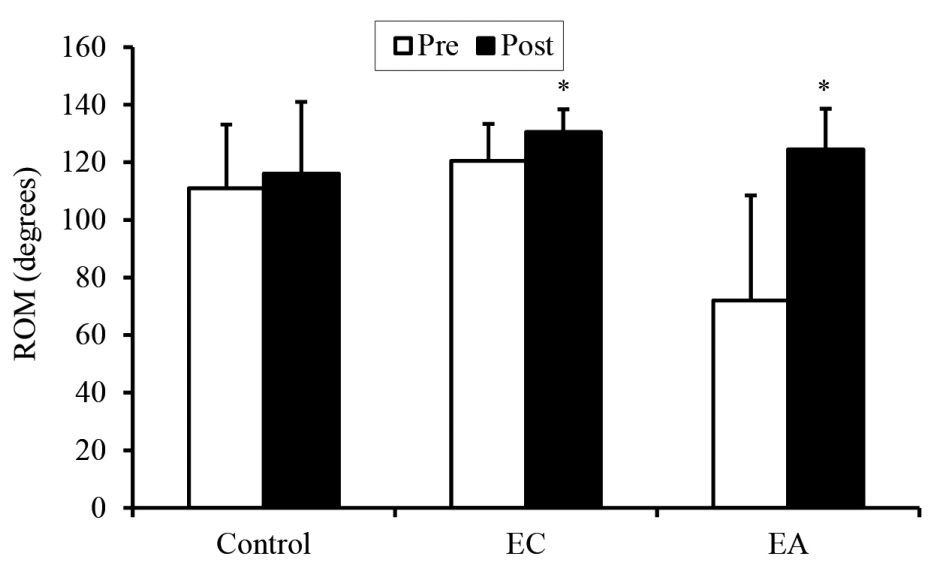

b

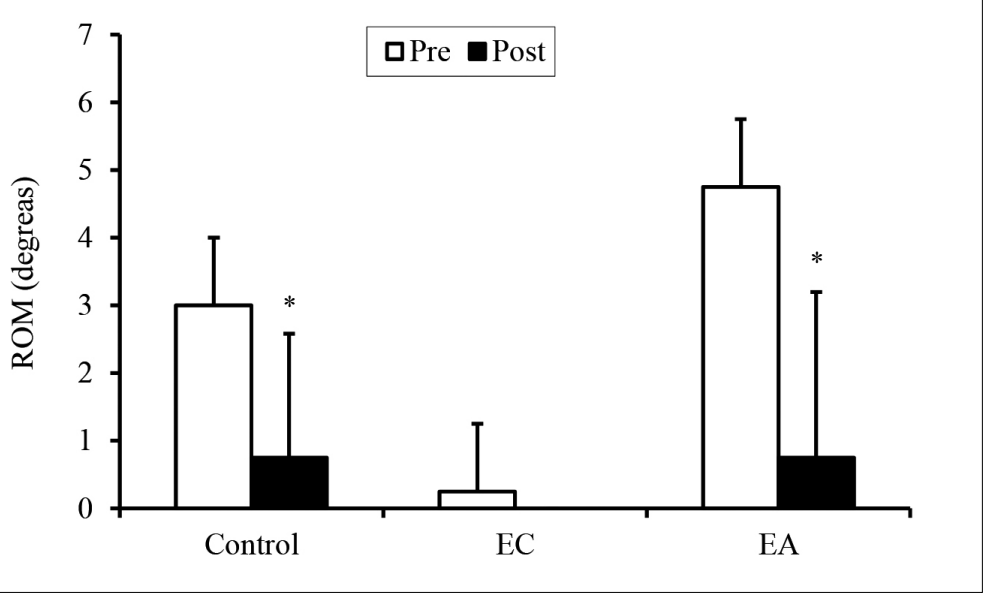

Range of motion. The initial values of knee extension in all groups (control, EC and EA) differed significantly (Figure 6; $p<.05$ ). The lowest value was in EC group, the highest value - in EA group. After wearing knee brace in control and EA groups, knee extension significantly increased $(p<.05)$. The highest pre-post increase was in EA subjects. Similar, the highest results increase were found for knee flexion in EA group $(p<.05)$ with no changes in control group $(p>.05)$.

Swelling. Knee joint average circumference post wearing the knee brace of control and EC group of subjects did not change, whereas EA group subjects' average volume of knee joint statistically significantly decreased in all measurements $(p<.05$; Figure 7). 

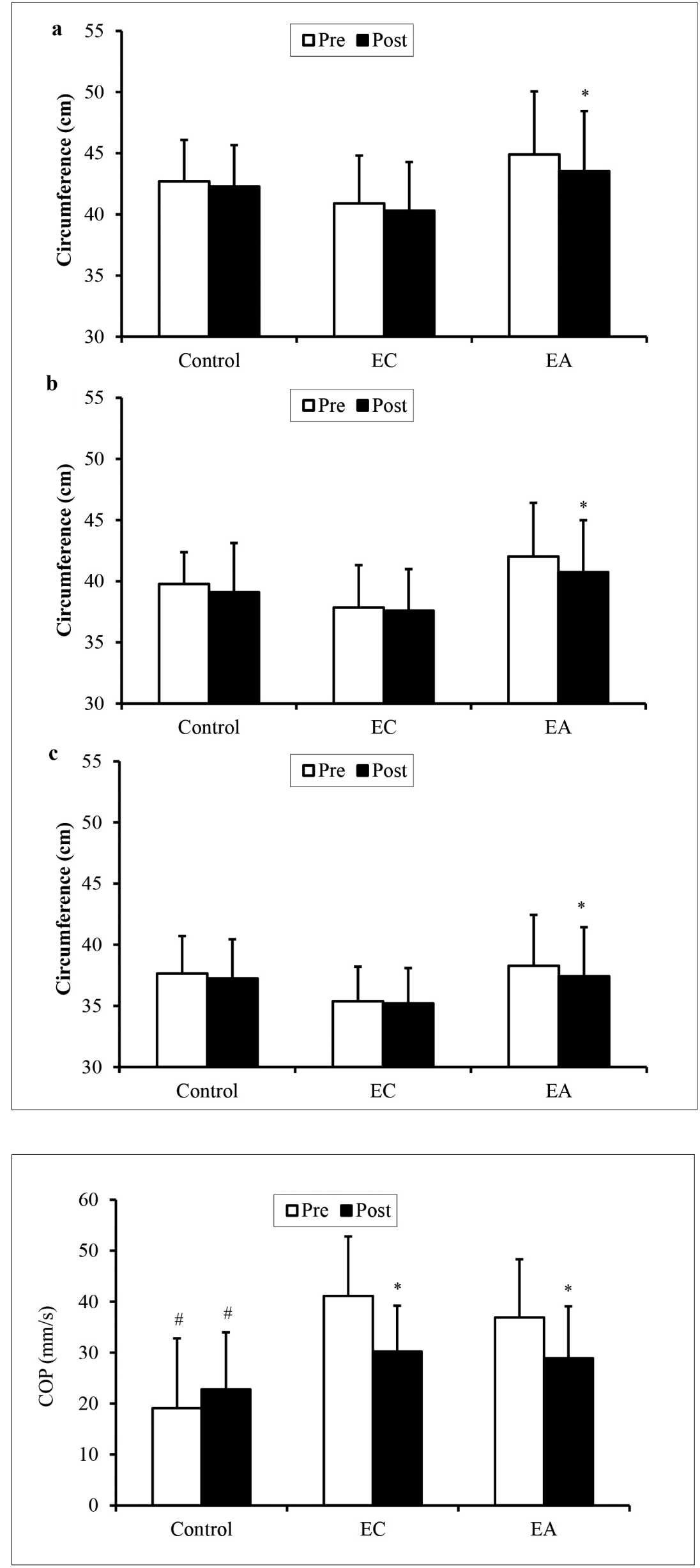

Figure 7. Mean values (coefficient of variation (CV)) of knee joint circumference (a) over the joint, (b) at the patella, (c) below the flange joint of Control, Experimental Chronic pain (EC) and Experimental Acute pain (EA) groups pre and post knee brace wear

Note. $* p<.05$ comparing to pre intervention value.

Figure 8. Mean values (coefficient of variation (CV)) of changes in the velocity of the centre of pressure (COP) of Control, Experimental Chronic pain (EC) and Experimental Acute pain (EA) groups pre and post knee brace wear

Note. $* p<.05$ comparing to pre intervention value, $\# p<.05$ compared with EC and EA groups. 
Balance. The results showed that subjects in EC and EA groups showed significantly lower changes in velocity of centre of pressure after intervention $(p<0.05$, Figure 8$)$. No changes in balance were recorded for control group $(p>.05)$.

\section{DISCUSSION}

Research results showed that low-intensity laser effect with the application of the immobilization is effective for the reduction of pain, relief of inflammatory processes, and rebuilding of patients' daily routine functions. The mobile innovative device is particularly effective in treating knee pain in the acute period.

Technical characteristics of our laser did not differ from lasers used in hospitals. The analysis shows that the diodes most commonly used in clinical practice are $808 \mathrm{~nm}$ LEDs with the greatest depth of penetration into the tissues, and the radiation power of up to several hundred milliwatts (Bjordal et al., 2008; van Middelkoop et al., 2011). In our case, infrared laser diodes of the same power incorporated in the brace, have reduced the patient's pain (especially in the acute condition), swelling in the calf and increased the range of flexion and extension of the knee. In addition, subjects subjectively better assessed their skills in everyday activities as measured by the Lysholm scale. Finally, clinical indicators improved more in the experimental group (especially in acute pain) than in the placebo group suggesting that the patient recovers faster using a portable (mobile) laser in the immobilization period. Our data are in accordance with other studies where positive effect of laser application were shown on humans and animals (Hegedus et al., 2009; Pallotta et al., 2012; Shen at al., 2009; Sushko, Lymans'kyi Iu, \& Huliar, 2007; Walsh et al., 2000). It is believed that the positive effect of complex treatment can be observed primarily due to the efficiency of laser improving microcirculation of the damaged tissue and promoting collagen synthesis (Hegedus et al., 2009; Rola et al., 2012).

Since balance control involves a multijoint system (ankle, knee, hip, etc.), any joint dysfunction may reduce the stability of balance (Turcot, Sagawa, Hoffmeyer, Suvà, \& Armand, 2015). Effect of laser therapy on postural stability has been very little studied. However, it has been established that the application of laser therapy statistically significantly reduced knee pain in patients suffering from osteoarthritis (Nakamura at al., 2014). The findings of our study confirmed the positive effects of rehabilitation with laser in the treatment of chronic and acute knee pain. Pain reduction improved performance in knee function thus improving balance stability of patients.

Despite the positive clinical results, knee extensor and flexor muscle contraction strength and proprioception indicator - muscle contraction strength variability during the period - changed slightly (except for isokinetic torque at $60 \%$ second speed). High variance of values of strength and proprioception test results showing the heterogeneity of the sample should be noted as well. The reason for the apparent discrepancy in muscle torque and clinical test results might reflect different intervention effects on very different mechanisms responsible for soreness, swelling, balance, strength and proprioception. Results support the notion that immobilization with brace and laser therapy cannot replace muscle strengthening program, especially in chronic conditions. It is likely that immobilization itself may prevent decrease in muscle force variability at low intensities because lack of movement does not stimulate muscle proprioception (Duchateau \& Enoka, 2008).

Probably the most important finding in present study was that in general the positive effect of interventions was evident applying the intervention at home rather than in a stationary clinic. In this case, treatment costs are reduced for research participants and rehabilitators spend less time. Yet in clinic, laser therapy is used only by qualified personnel because the procedure requires specific knowledge about the diversity of exposed points (painful area, active points, etc.). Research results showed that after patients have been trained to use a low-frequency laser in outpatient (home) conditions, good results can be achieved. In pain patients have a high motivation to learn to use a brace with laser and use the interventions according to the given instructions regularly. We have observed that patients had difficulty applying the laser therapy at home because the laser beam coverage was low, and this made it hard trying to hit exactly the painful areas. Further improving the knee brace it would be appropriate to increase the laser impact zone thus decreasing the influence of patient's low qualification factor (error probability) for the final result.

In conclusion, knee brace with laser therapy in the rehabilitation period ensures faster healing of 
patients, particularly in acute pain, while muscle strength and proprioception remains depressed. Further studies are necessary to search for optimal laser parameters to make it simpler to use at home with laser impact area expansion being attractive candidate for such purposes.

\section{REFERENCES}

Bjordal, J. M., Couppé, C., Chow, R. T., Tunér, J., \& Ljunggren, E. A. (2003). A systematic review of low level laser therapy with location-specific doses for pain from chronic joint disorders. Australian Journal of Physiotherapy, 49(2), 107-16.

Bjordal, J. M., Lopes-Martins, R. A., Joensen, J., Couppe, C., Ljunggren, A. E., Stergioulas, A., \& Johnson, M. I. (2008). A systematic review with procedural assessments and meta-analysis of low level laser therapy in lateral elbow tendinopathy (tennis elbow). BMC Musculoskeletal Disorders, 9, 75. doi:10.1186/1471-2474-9-75

Bollen, S. (2000). Injuries of the sporting knee. Epidemiology of knee injuries: Diagnosis and triage. British Journal of Sports Medicine, 34, 227-228.

Duchateau, J., \& Enoka, R. M. (2008. Neural control of shortening and lengthening contractions: Influence of task constraints. Journal of Physiology, 586(24), 58535864. doi: 10.1113/jphysiol.2008.160747

Hegedus, B., Viharos, L., Gervain, M., \& Gálfi, M. (2009). The effect of low-level laser in knee osteoarthritis: A double-blind, randomized, placebo-controlled trial. Photomedicine and Laser Surgery, 4(27), 577-584. doi: 10.1089/pho.2008.2297

Jang, H., \& Lee, H. (2012) Meta-analysis of pain relief effects by laser irradiation on joint areas. Photomedicine and Laser Surgery, 30(8), 405-417.

Miyama, M., \& Nosaka, K. (2007). Protection against muscle damage following fifty drop jumps conferred by ten drop jumps. Journal of Strength and Conditioning Research, 21(4), 1087-1092.

Nakamura, T., Ebihara, S., Ohkuni, I., Izukura, H., Harada, T., Ushigome, N., ... Kubota, A. (2014). Low Level Laser Therapy for chronic knee joint pain patients. Laser Therapy, 23(4), 273-277.

Pallotta, R. C., Bjordal, J. M., Frigo, L., Leal Junior, E. C., Teixeira, S., Marcos, R. L., ... Lopes-Martins, R. A. (2012). Infrared (810-nm) low-level laser therapy on rat experimental knee inflammation. Lasers in Medical Science, 27, 71-78.

Peter, W. F. H., Jansen, M. J., Hurkmans, E. J., Bloo, H., Dekker-Bakker, L. M. M. C. J., Dilling ... Vliet
Vlieland, T. P. M. (2011). Physiotherapy in hip and knee osteoarthritis: Development of a practice guideline concerning initial assessment, treatment and evaluation. Acta Reumatológica Portuguesa, 36, 268-281.

Rola, P., Doroszko, A., \& Derkacz, A. (2014) The Use of Low-Level Energy Laser Radiation in Basic and Clinical Research. Advances in Clinical and Experimental Medicine, 23(5), 835-842.

Shen, X., Zhao, L., Ding, G., Gao, J., Wang, L., \& Lao, L. (2009) Effect of combined laser acupuncture on knee osteoarhritis: A pilot study. Lasers in Medical Science, 24(2), 129-136.

Skurvydas, A., Masiulis, N., Gudas, R., Dargevičiūtè, G., Parulyte, D., Trumpickas, V., \& Kalesinskas, J. R. (2011) Extension and flexion torque variability in ACL deficiency. Knee Surgery, Sports Traumatology, Arthroscopy: Official Journal of the ESSKA, 19(8), 1307-1313.

Sushko, B. S., Lymans'kyi Iu, P., \& Huliar, S. O. (2007). Action of the red and infrared electromagnetic waves of light-emitting diodes on the behavioural manifestation of somatic pain. Fiziolohichnyi Zhurnal, 53(3), 51-60.

Tegner, Y., \& Lysholm, J. (1985). Rating systems in the evaluation of knee ligament injuries. Clinical Orthopaedics and Related Research, 198, 43-49.

Turcot, K., Sagawa, Y. Jr., Hoffmeyer, P., Suvà, D., \& Armand, S. (2015). Multi-joint postural behavior in patients with knee osteoarthritis. The Knee. pii: S09680160(14)00220-8. doi: 10.1016/j.knee.2014.09.001. [Epub ahead of print]

Van Middelkoop, M., Rubinstein, S. M., Kuijpers, T., Verhagen, A. P., Ostelo, R., Koes, B. W., \& van Tulder, M. W. (2011). A systematic review on the effectiveness of physical and rehabilitation interventions for chronic non-specific low back pain. European Spine Journal, 20, 19-39. doi: 10.1007/s00586-010-1518-3

Walsh, D. M., Baxter, G. D., \& Allen, J. M. (2000). Lack of effect of pulsed low-intensity infrared $(820 \mathrm{~nm})$ laser irradiation on nerve conduction in the human superficial radial nerve. Lasers in Surgery and Medicine, 26(5), 485-490. 